\title{
The Therapeutic Effects and Mechanisms of Quercetin on Metabolic Diseases: Pharmacological Data and Clinical Evidence
}

\author{
Huan Yi, ${ }^{1}$ Hengyang Peng, ${ }^{2}$ Xinyue Wu, ${ }^{1}$ Xinmei Xu, ${ }^{1}$ Tingting Kuang, ${ }^{3}$ Jing Zhang, ${ }^{3}$ \\ Leilei Du ${ }^{(D)}{ }^{3}$ and Gang Fan $(\mathbb{D})^{1,3}$ \\ ${ }^{1}$ State Key Laboratory of Southwestern Chinese Medicine Resources, School of Pharmacy, Chengdu University of Traditional \\ Chinese Medicine, Chengdu 611137, China \\ ${ }^{2}$ Animal Health Research Institute, Tongwei Co., Ltd., Chengdu 610041, China \\ ${ }^{3}$ School of Ethnic Medicine, Chengdu University of Traditional Chinese Medicine, Chengdu 611137, China
}

Correspondence should be addressed to Leilei Du; duleilei@cdutcm.edu.cn and Gang Fan; fangang1111@163.com

Received 19 November 2020; Revised 17 January 2021; Accepted 8 June 2021; Published 24 June 2021

Academic Editor: David Nieman

Copyright ( $\odot 2021$ Huan Yi et al. This is an open access article distributed under the Creative Commons Attribution License, which permits unrestricted use, distribution, and reproduction in any medium, provided the original work is properly cited.

\begin{abstract}
Metabolic diseases have become major public health issues worldwide. Searching for effective drugs for treating metabolic diseases from natural compounds has attracted increasing attention. Quercetin, an important natural flavonoid, is extensively present in fruits, vegetables, and medicinal plants. Due to its potentially beneficial effects on human health, quercetin has become the focus of medicinal attention. In this review, we provide a timely and comprehensive summary of the pharmacological advances and clinical data of quercetin in the treatment of three metabolic diseases, including diabetes, hyperlipidemia, and nonalcoholic fatty liver disease (NAFLD). Accumulating evidences obtained from animal experiments prove that quercetin has beneficial effects on these three diseases. It can promote insulin secretion, improve insulin resistance, lower blood lipid levels, inhibit inflammation and oxidative stress, alleviate hepatic lipid accumulation, and regulate gut microbiota disorders in animal models. However, human clinical studies on the effects of quercetin in diabetes, hyperlipidemia, and NAFLD remain scarce. More clinical trials with larger sample sizes and longer trial durations are needed to verify its true effectiveness in human subjects. Moreover, another important issue that needs to be resolved in future research is to improve the bioavailability of quercetin. This review may provide valuable information for the basic research, drug development, and clinical application of quercetin in the treatment of metabolic diseases.
\end{abstract}

\section{Introduction}

Metabolic diseases encompass a constellation of maladies characterized by disruption of normal metabolism, involving the processing or transport of proteins (amino acids), carbohydrates (sugars and starches), or lipids (fatty acids) [1]. Diabetes is a kind of common endocrine metabolic diseases. According to the latest report from the International Diabetes Federation, about 463 million adults (20-79 years old) worldwide were suffering from diabetes, and the number of diabetic patients in China ranked first [2]. It is reported that metformin combined with lifestyle interventions can prolong the onset of diabetes [3]. However, treatment with metformin may present toxic side effects, such as causing gastrointestinal discomfort and even lactic acidosis. Hyperlipidemia, also known as dys- lipidemia, is a metabolic disease characterized by excess lipids in the blood plasma. A high-fat diet (HFD) or poloxamer-407 (P-407) can cause abnormal lipoprotein metabolism and lead to hyperlipidemia, which is characterized by elevated triglycerides (TG), total cholesterol (TC), low-density lipoprotein (LDL), and very low-density lipoprotein (VLDL), while the level of high-density lipoprotein (HDL) is decreased [4]. Among lipid-lowering drugs, statins and resins are mainly used to reduce TC and LDL, while niacin is used to reduce TG. The most common side effects of simvastatin are muscle pain and gastrointestinal symptoms [5]. Nonalcoholic fatty liver disease (NAFLD) refers to a metabolic disease in which the lipid content in the liver exceeds 5 10\%, and its etiology has nothing to do with alcohol, drugs, and genetics [6]. The prevalence of NAFLD in ordinary adults is $10 \%$ 30\%. 
Pioglitazone can be used to treat NAFLD, yet with possible side effects such as weight gain and hypoglycemia [7].

Due to the acceleration of population aging and changes in behavior and socioeconomics, metabolic diseases have become a serious public health problem. Therefore, there is an urgent need to seek safe and effective therapeutic drugs. Phytotherapy has been valued in diverse traditional cultures and it is believed that natural products are more economical and safer than chemical products [8]. Because of their therapeutic properties and safety, natural compounds from vegetables, fruits, or medicinal plants are widely used in the prevention or/and treatment of diseases. As a super family of phytochemicals, flavonoids extracted from natural plants are reported to have potential therapeutic value for metabolic diseases [9]. Quercetin is an important flavonoid compound found in many edible and medicinal plants. In recent years, quercetin has received widespread attention due to its good potential in the treatment of metabolic diseases. It has a variety of pharmacological activities, such as hypoglycemic, hypolipidemic, cardiovascular protecting, anti-inflammatory, anticancer, and hepatoprotective effects [10-12]. Some clinical trials have also reported its beneficial effects on type 2 diabetes mellitus (T2DM), hyperlipidemia, and NAFLD [13-15]. However, most of these reports are scattered and lack a systematic summary.

In this review, the natural sources and physicochemical properties of quercetin were introduced. Its absorption, metabolism, and bioavailability were also outlined. More importantly, we provide a timely and comprehensive overview of the pharmacological advances and clinical evidence of quercetin in the treatment of three metabolic diseases (diabetes, hyperlipidemia, and NAFLD). The information may provide an important reference for the future research and development of quercetin.

\section{Methods}

In this review, we performed a comprehensive literature search using some online databases (e.g., ISI Web of Science, PubMed, Science Direct, Google Scholar, and China National Knowledge Infrastructure) to obtain information on the effects of quercetin on three metabolic diseases. In the search process, the following keywords were used: "quercetin" and "metabolic diseases", "diabetes", "hyperlipidemia", or "nonalcoholic fatty liver disease". The references of all retrieved articles were also reviewed to identify other articles that may be missed using the above search terms. After the literature search, we screened the titles and abstracts of the extracted studies and further examined the full text of the included articles to confirm eligibility for inclusion in this review. Editorials, meeting abstracts, and studies with incorrect, incomplete, or unavailable data were excluded.

\section{Natural Sources and Physicochemical Properties of Quercetin}

3.1. Natural Sources. As one of the most abundant dietary flavonoids in the human diet to date, quercetin is widely distributed in vegetables and fruits. Xiao et al. corrected the previous misconception and what they believed is that quercetin intake accounted for about $60 \%-75 \%$ of total flavonols, not total dietary flavonoids [16]. The sources of quercetin differ in different countries and regions due to the diversity of regional environment, traditional culture, and other factors. In Chinese population, the main dietary sources of quercetin were green vegetables, tubers, tomatoes, oranges, apples, green tea, pears, sweet potato, and black tea [13]. In Japan, quercetin was mainly provided by green tea and onion in summer and winter. In the meanwhile, some summer vegetables, such as green pepper, asparagus, red lettuce, and tomato, were also good sources of quercetin [17]. In Australian adults, apples, black tea, grapes, lettuce, green beans, tomatoes, mature onions, green tea, coffee, and wine were the major sources of quercetin [18]. Häkkinen et al. reported that the main sources of quercetin were berry, apple, tea, and onion in Finland [19]. Table 1 lists the natural sources of quercetin [20-38].

Moreover, quercetin can also be obtained from traditional medicinal plants. It is widely distributed in the roots, leaf, and fruit of many medicinal plants, such as Petroselinum crispum (Mill.) Fuss (Apiaceae), Nepeta cataria L. (Labiatae), Ginkgo biloba L. (Ginkgoaceae), Mentha canadensis L. (Labiatae), Polygonum orientale L. (Polygonaceae), and Crataegus pinnatifida Bunge (Rosaceae). High-performance liquid chromatography method is commonly used to determine the content of quercetin in herbal medicines. The content of quercetin varies greatly in different plants, ranging from $0.02 \mathrm{mg} / \mathrm{g}$ to $63.60 \mathrm{mg} / \mathrm{g}$. Among the reported plants, $P$. orientale has the highest concentration of quercetin (63.60 mg/g), followed by G. biloba $(33.00 \mathrm{mg} / \mathrm{g})$ and $M$. canadensis $(10.80 \mathrm{mg} / \mathrm{g})$.

3.2. Physical and Chemical Properties of Quercetin. Quercetin is a bright yellow needle-like crystal with a melting point of $313-314^{\circ} \mathrm{C}$. Its molecular formula is $\mathrm{C}_{15} \mathrm{H}_{10} \mathrm{O}_{7}$ and molecular mass is 302.23. Quercetin is also known as 3,3', $4^{\prime}, 5,7$-pentahydroxyflavone or $3,3^{\prime}, 4^{\prime}, 5,7$-pentahydroxy-2-phenylchromen-4-one (Figure 1). The basic carbon chain of quercetin is $\mathrm{C6}-\mathrm{C} 3-\mathrm{C} 6$, which consists of two benzene rings (A and $\mathrm{B})$ and an oxygen-containing heterocycle $(\mathrm{C})$. There is a hydroxyl group at the $3,3^{\prime}, 4^{\prime}, 5$, and 7 positions, respectively. The molecular structure of quercetin is known as the best configuration for scavenging free radicals and binding transition metal ions, so it is considered to have a potent antioxidant effect [39]. Chemical stability of quercetin is affected by many factors, such as $\mathrm{pH}$, temperature, storage time, and the presence of oxygen and metal ions [40]. Quercetin is unstable in organic solutions with a $\mathrm{pH}$ greater than 7 , which may be due to its central ring structure. Moreover, quercetin is a common flavonoid aglycone, so it is soluble in ethanol, methanol, and ethyl acetate, hardly soluble in petroleum ether, benzene, ether, and chloroform, and almost insoluble in water.

\section{Bioavailability of Quercetin}

The bioavailability of quercetin is closely related to its biological activity and health benefits. In view of the wide 


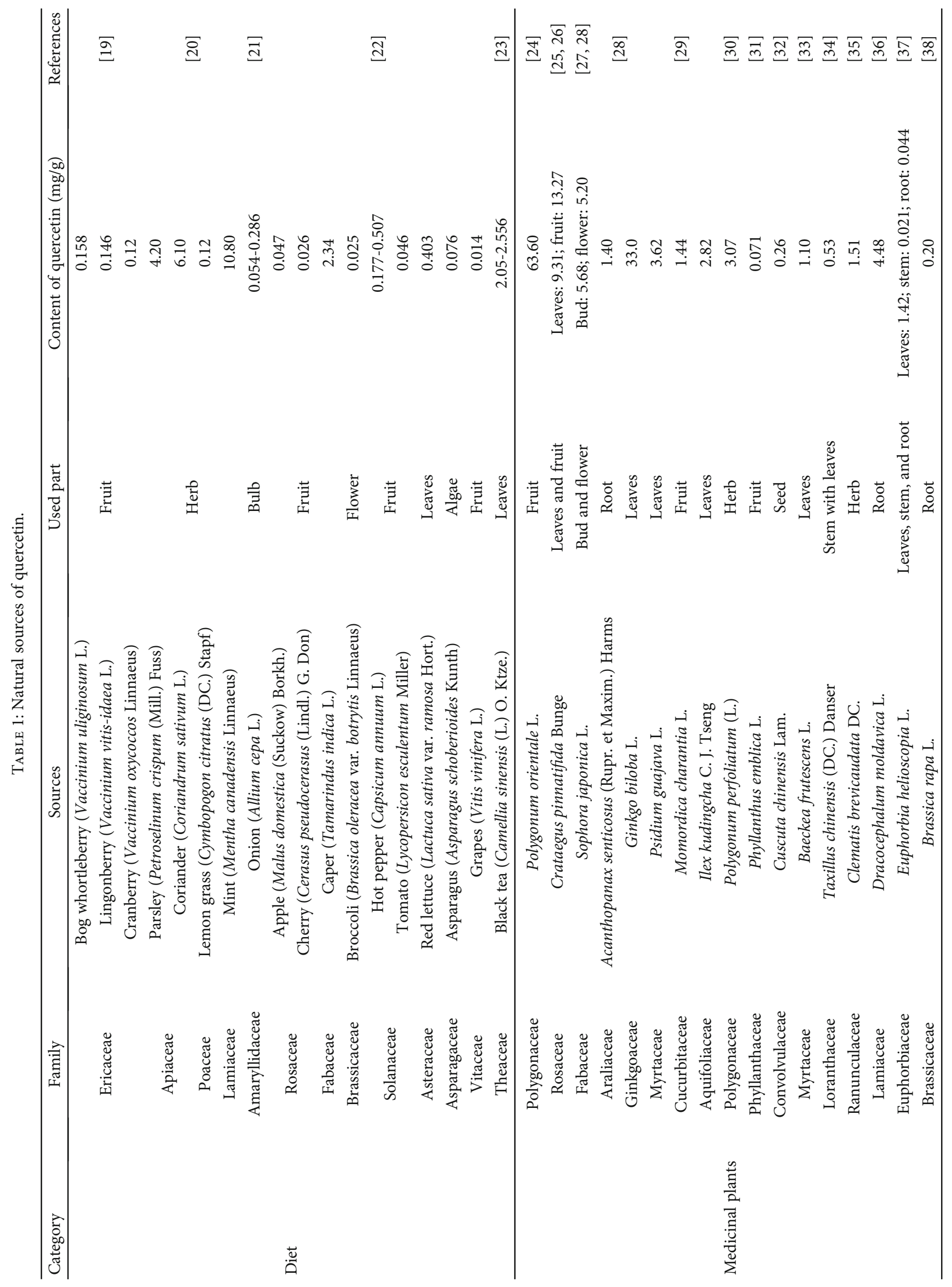




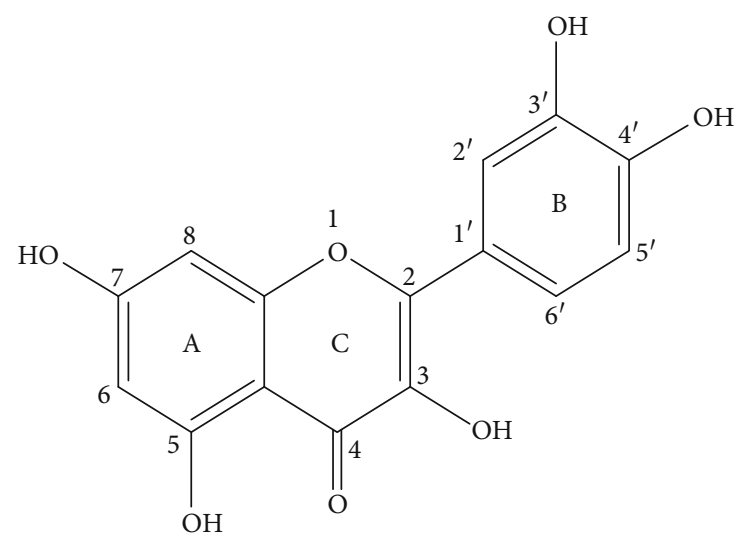

FIGURE 1: The chemical structure of quercetin.

application of quercetin-containing products and the drugforming potential of quercetin, its bioavailability should be carefully evaluated. Previous investigations have shown that quercetin has poor and highly variable bioavailability [41], which may be related to its pharmacokinetic properties. Absorption is an important factor affecting the oral bioavailability of quercetin. Pharmacokinetic studies suggest that quercetin has low absorption [41]. Chen et al. found that after oral administration, only $6.7 \%$ of quercetin was absorbed into the portal vein, while about $93 \%$ was lost in the intestine [42]. However, quercetin derivatives, especially its glycosides, glucuronide, or sulfate conjugates, are more easily absorbed than quercetin $[42,43]$. In the intestine, quercetin could be degraded by some intestinal bacteria (e.g., Eubacterium oxidoreducens, Clostridium orbiscindens, and Eubacterium ramulus), with C ring fission and dehydroxylation to produce lower molecular weight phenolic compounds, which were easily absorbed [44]. Moreover, quercetin could be glucuronidated by uridine diphosphate glucuronosyltransferase and methylated by catechol-Omethyltransferase in the small intestine. Subsequently, they were transported to the liver through the portal circulation to be further metabolized, involving not only Omethylation and glucuronidation, but also sulfonylation by sulfotransferases [45]. Chen et al. found that approximately $93.3 \%$ of quercetin was metabolized in the intestine and only $3.1 \%$ was metabolized in the liver in rats [42]. The absorbed quercetin and its derivatives are mainly excreted into bile or urine and eventually eliminated in feces [40]. Quercetin bioavailability may be affected by its hepatic biliary excretion [41]. In addition, there are obvious individual differences in the bioavailability of quercetin, which is related to some endogenous (e.g., gender, age, and intestinal permeability) and exogenous factors (e.g., food matrix, dietary fat, and glycosylation of quercetin) $[41,46]$. Besides, the duration and frequency of medication also affect the plasma concentration of quercetin. Some studies indicated that long-term or repeated supplement of quercetin could increase its bioavailability [40,47]. The above findings suggest that a variety of factors can affect the oral bioavailability of quercetin. There is no doubt that improving the bioavailability of quercetin and its metabolites is expected to enhance its biological activity and health benefits.

\section{Pharmacological Effects and Molecular Mechanisms of Quercetin against Three Metabolic Diseases}

5.1. Diabetes. Accumulating evidences indicate that quercetin has good antidiabetic effects. Quercetin may exert antidiabetic effects through various mechanisms, including promoting insulin secretion, improving insulin resistance, maintaining glucose homeostasis, and inhibiting inflammation, oxidative stress, and apoptosis. The main targets modulated by quercetin are shown in Figure 2.

5.1.1. Quercetin Promotes Insulin Secretion. Healthy individual maintains a stable blood glucose level through basal insulin secretion, while diabetic hyperglycemia is the main symptom caused by insulin deficiency. Bhattacharya et al. found that quercetin had the potential to promote glucosestimulated insulin secretion and insulin expression in INS$1 \mathrm{E}$ (insulin-secreting rat insulinoma) cells [48]. A study reported that quercetin could resist cholesterol-induced pancreatic $\beta$ cell dysfunction, thereby maintaining glucosestimulated insulin secretion and glycemic control [49]. Another study found that quercetin was able to dosedependently improve insulin secretion and reduce blood glucose levels after 6 weeks of oral administration in diabetic rats [50]. In addition, quercetin could accelerate the recovery of $\beta$ cells by increasing the expression of vascular endothelial growth factor (VEGF) and its receptor VEGFR2 in the pancreas of diabetic rats [50]. Youl et al. found that quercetin could significantly enhance the insulin secretion of INS-1 pancreatic $\beta$ cells by specifically activating extracellular signal-regulated kinase 1/2 (ERK1/2) [51]. Moreover, Bardy et al., Zhuang et al., and Kittl et al. found that quercetin significantly stimulated insulin secretion by activating the intracellular $\mathrm{Ca}^{2+}$ signaling pathway [52-54].

5.1.2. Quercetin Improves Insulin Resistance. El-Baky reported that quercetin could decrease insulin resistance evaluated by homeostasis model assessment (HOMA) in diabetic rats [55]. Shao et al. found that quercetin significantly reduced fasting glucose and increased insulin sensitivity index, causing significant changes in the insulin level of diabetic rats [56]. Gaballah et al. discovered that quercetin treatment could significantly improve insulin resistance in HFD and streptozotocin- (STZ-) induced type 2 diabetic rats through the alleviation of pancreatic endoplasmic reticulum (ER) stress and oxidative stress, as well as counteraction of inflammation and $\beta$ cell death [57]. Chuang et al. found that quercetin attenuated the tumor necrosis factor- $\alpha$ - (TNF- $\alpha-$ ) mediated insulin resistance in primary human adipocytes by blocking the serine phosphorylation of insulin receptor substrate-1 (IRS-1) and the expression of phosphatase(PTP-) 1B gene [58]. In addition, quercetin affected the hypothalamic insulin signaling pathway by upregulating the phosphorylation of insulin receptors (InsRs) and protein kinase (PK) B, also known as Akt, thereby effectively 


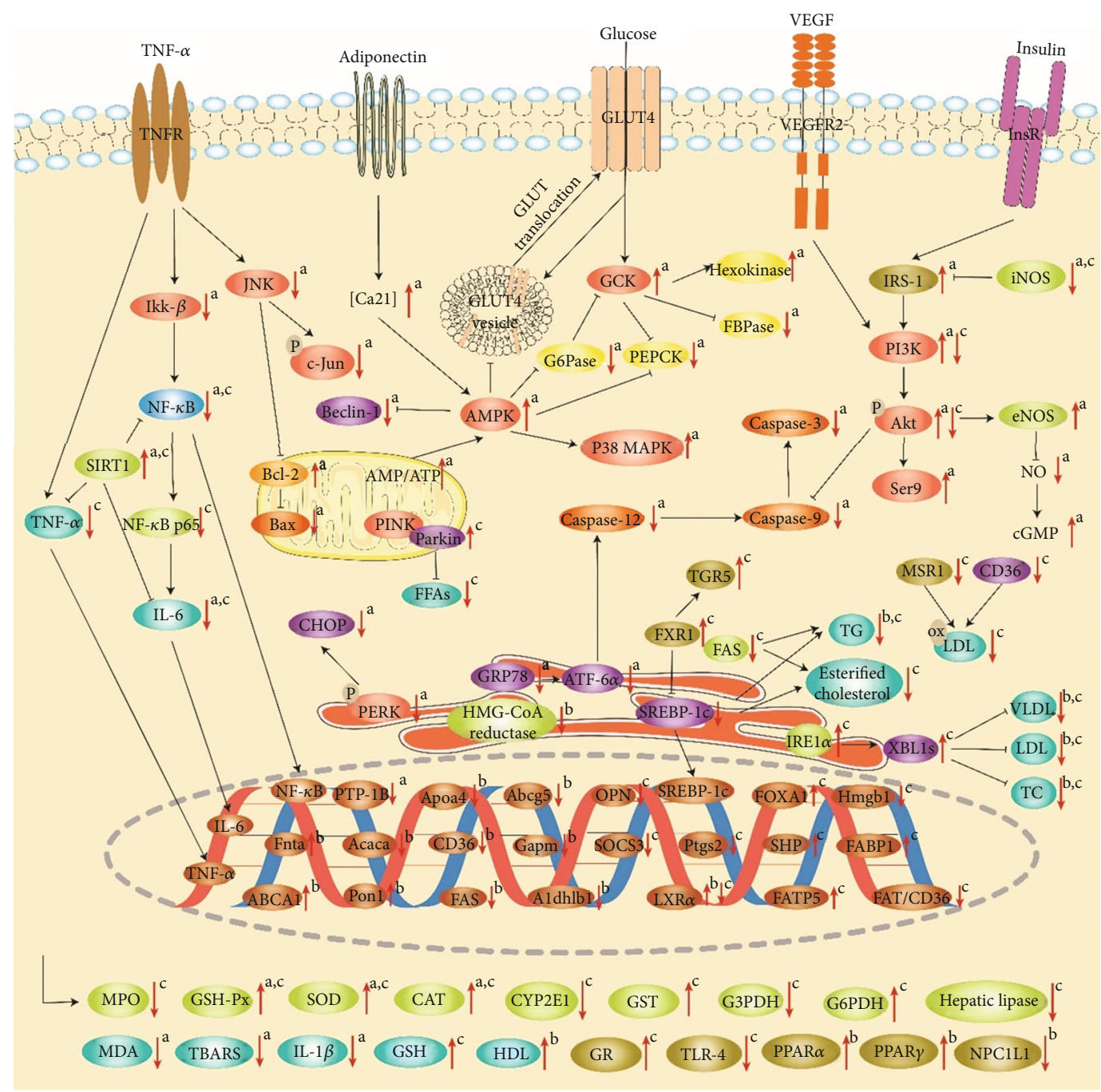

FIGURE 2: Quercetin can improve diabetes, hyperlipidemia, and NAFLD by modulating the marked targets. $\uparrow$ indicates increase and $\downarrow$ indicates decrease; $\rightarrow$ indicates stimulatory effect and $\dashv$ indicates inhibitory effect. In the upper right corner, a represents the effect of quercetin on diabetes, $b$ represents the effect of quercetin on hyperlipidemia, and c represents the effect of quercetin on NAFLD. ABCA1: ATP-binding cassette transporter A1; Abcg5: ATP-binding cassette subfamily G member 5; Acaca: acetyl-coenzyme A carboxylase $\alpha$; Akt: protein kinase B; Aldh1b1: aldehyde dehydrogenase 1 family member B1; AMPK: adenosine monophosphate-activated protein kinase; Apoa4: apolipoprotein A-IV; ATF-6 $\alpha$ : activating transcriptional factor $6 \alpha$; CAT: catalase; CD36: cluster of differentiation 36; cGMP: cyclic 3,5-guanosine monophosphate; CHOP: CCAAT/enhancer-binding protein homologous protein; CYP2E1: cytochrome P450 2E1; eNOS: endothelial nitric oxide synthase; FABP1: fatty acid-binding protein 1; FAS: fatty acid synthase; FAT/CD36: fatty acid translocase CD36; FATP5: fatty acid transport protein 5; FFAs: free fatty acids; FOXA1: forkhead box protein A1; FXR1: farnesoid X receptor 1; FAS: fatty acid synthase; FBPase: fructose-bisphosphatase; Fnta: farnesyltransferase CAAX box $\alpha$; G6Pase: glucose-6-phosphate; G3PDH: glycerol-3phosphate dehydrogenase; G6PDH: glucose-6-phosphate dehydrogenase; GCK: glucokinase; GLUT4: glucose transporter type 4; Gpam: glycerol-3-phosphate acyltransferase mitochondrial; GR: glutathione reductase; GRP78: glucose regulated protein 78; GSH: glutathione; GSH-Px: glutathione peroxidase; GST: glutathione-S-transferase; HDL: high-density lipoprotein; Hmgb1: high-mobility group box 1; HMG-CoA reductase: 3 -hydroxy-3-methylglutaryl-CoA reductase; Ikk- $\beta$ : inhibitor $\kappa \mathrm{B}$ kinase $\beta$; IL- $1 \beta$ : interleukin- $1 \beta$; IL-6: interleukin-6; iNOS: inducible nitric oxide synthase; InsR: insulin receptor; IRE1 $\alpha$ : inositol-requiring transmembrane kinase/endoribonuclease $1 \alpha$; IRS-1: insulin receptor substrate-1; JNK: c-Jun- $\mathrm{NH}_{2}$ terminal kinase; LDL: low-density lipoprotein; LXR $\alpha$ : liver X receptor $\alpha$; MDA: malondialdehyde; MPO: myeloperoxidase; MSR1: macrophage scavenger receptor 1; NF- $\kappa$ B: nuclear factor- $\kappa$ B; NO: nitric oxide; NPC1L1: Niemann-Pick C1-like 1; OPN: osteopontin; ox-LDL: oxidized low-density lipoprotein; P38 MAPK: P38 mitogen-activated protein kinases; PEPCK: phosphoenolpyruvate carboxylase; PERK: protein kinase-like endoplasmic reticulum kinase; PI3K: phosphatidyl inositol 3 kinase; Pon1: paraoxonase1; PPAR $\alpha$ : peroxisome proliferator-activated receptor $\alpha$; PPAR $\gamma$ : peroxisome proliferator-activated receptor $\gamma$; Ptgs2: cyclooxygenase-2; PTP-1B: phosphatase-1B; Ser9: phosphorylated glycogen synthase kinase $3 \beta$; SHP: small heterodimer partner; SIRT1: sirtuin 1; SOCS3: suppressor of cytokine signaling 3; SOD: superoxide dismutase; SREBP-1c: sterol regulatory element-binding protein-1c; TBARS: thiobarbituric acid-reactive substances; TC: total cholesterol; TG: triglyceride; TGR5: Takeda G protein-coupled receptor 5; TLR-4: Toll-like receptor-4; TNFR: tumor necrosis factor receptor; TNF- $\alpha$ : tumor necrosis factor- $\alpha$; VEGF: vascular endothelial growth factor; VEGFR2: vascular endothelial growth factor receptor 2; VLDL: very low-density lipoprotein; XBP1s: X-boxbinding protein 1 . 
improving insulin resistance in high fructose-induced rats [59]. Moreover, Dhanya et al. found that the adenosine monophosphate-activated protein kinase (AMPK) signaling pathway could help quercetin correct insulin resistance by bypassing the GLUT4 translocation of the insulin regulatory system in L6 myotubes [60].

5.1.3. Quercetin Maintains Glucose Homeostasis. Diabetes patients often have disorders of glucose metabolism. Sandeep and Nandini found that quercetin treatment restored the impaired protein expression of key insulin signaling molecules, such as IRS-1 and phosphatidyl inositol 3 kinase $(\mathrm{PI} 3 \mathrm{~K})$, thereby promoting insulin-mediated glucose uptake in the brain of STZ-induced diabetic rats [61]. In the liver of rats, quercetin induced AMPK activation, which reduced glucose production mainly by downregulating key glycogenic isoenzymes, such as glucose-6-phosphate (G6Pase) and phosphoenolpyruvate carboxylase (PEPCK) [62, 63]. Peng et al. found that quercetin significantly enhanced the expression of phosphorylated glycogen synthase kinase $3 \beta$ (GSK$3 \beta$ ), namely, Ser9, and Akt phosphorylation promoted the expression of glucokinase (GCK) protein (known as hexokinase type IV) in the liver of diabetic rats so as to enhance the synthesis of hepatic glycogen and alleviate the glucose metabolism disorders [64]. The research of Eid et al. and Alam et al. shows that quercetin increases glucose uptake by stimulating the translocation of the glucose transporter type 4 (GLUT4) to the plasma membrane in murine skeletal muscle cells $[62,65]$. Dhanya et al. and Eid et al. used PK inhibitors to demonstrate that quercetin exerted its role in ameliorating glucose uptake in L6 myotubes through the adenosine AMPK pathway and its downstream target, P38 mitogenactivated protein kinases (MAPK) $[60,66]$. Peng et al. and Xiao et al. reported that quercetin could promote glucose processing in adipocytes and liver by regulating the activity of sirtuin 1 (SIRT1) [64, 67]. Alam et al. and Vessal et al. found that the activity of hexokinase was increased, while G6Pase and fructose-bisphosphatase (FBPase) activities were decreased in quercetin-treated diabetic mice and rats $[65,68]$. These results indicated that quercetin could reduce gluconeogenesis and increase glycolysis, ultimately reducing hyperglycemia and altering glucose metabolism disorders $[65,68]$. In addition, quercetin, as an inhibitor of carbohydrate hydrolases, such as pancreatic $\alpha$-amylase and intestinal $\alpha$-glucosidase, could slow down the hydrolysis of starch, thereby reducing the rate of glucose absorption and leading to a delayed rise in postprandial hyperglycemia $[69,70]$.

5.1.4. Quercetin Inhibits Inflammation in Diabetic Animals. Quercetin has been reported to have obvious antiinflammatory effects. Rifaai et al. found that inflammatory cell infiltration could be reduced by quercetin in pancreatic islets of STZ-induced diabetic rats [71]. Eitah et al. and Rivera et al. found that the anti-inflammatory effect of quercetin could be produced by increasing plasma adiponectin and reducing plasma nitrate plus nitrite (NOx) and TNF- $\alpha$ concentrations in diabetic rats $[72,73]$. In primary human adipocytes, quercetin could reduce TNF- $\alpha$-mediated inflam- mation [58]. It could directly inhibit the activation of ERK, cJun- $\mathrm{NH}_{2}$ terminal kinase (JNK), c-Jun, and nuclear factor- $\kappa \mathrm{B}$ $(\mathrm{NF}-\kappa \mathrm{B})$, which induced inflammatory gene expression and protein secretion, and indirectly activate peroxisome proliferator-activated receptor $\gamma(\operatorname{PPAR} \gamma)$ activity [58]. Similarly, quercetin significantly decreased the serum concentrations of some proinflammatory mediators, such as interleukin-6 (IL-6), in women with T2DM [74]. In another study, the levels of inhibitor $\kappa \mathrm{B}$ kinase $\beta$ (Ikk- $\beta$ ), TNF- $\alpha$, and IL- $1 \beta$ were decreased, and the expressions of Ikk- $\beta$ and TNF- $\alpha$ protein were also reduced in the heart of diabetic rats after quercetin treatment [75].

5.1.5. Quercetin Inhibits Oxidative Stress in Diabetic Animals. Oxidative stress has a potential role in the pathogenesis of diabetes. Fedosova et al. found that quercetin could reduce the oxidative function of polymorphonuclear neutrophils (PMN) in patients with non-insulin-dependent diabetes mellitus (NIDDM) [76]. Quercetin exerted its antioxidant effects by directly inhibiting the production of lipid peroxides, such as malondialdehyde (MDA) and thiobarbituric acid-reactive substances (TBARS), and indirectly promoting the production of endogenous antioxidants, such as antioxidant enzymes glutathione peroxidase (GSH-Px), superoxide dismutase (SOD), and catalase (CAT), in STZ-induced diabetic rats and $\mathrm{db} / \mathrm{db}$ mice $[55,75,77-85]$. Dokumacioglu et al. and Chis et al. reported that quercetin could ameliorate oxidative stress in STZ-induced diabetic rats through scavenging oxygen free radicals $[82,84]$. Iskender et al. found that quercetin inhibited oxidative damage by increasing SIRT1 level and decreasing NF- $\kappa \mathrm{B}$ level in STZ-induced diabetic rats [85]. Moreover, quercetin could prevent oxidative stress in liver tissues of STZ-induced diabetic rats by inhibiting the apoptosis process of target cells [86]. Quercetin could significantly reduce inducible nitric oxide synthase (iNOS) activity and nitric oxide (NO) concentration in the serum of diabetic mice [87]. In addition, quercetin could also protect the function and viability of INS-1 $\beta$ cells from oxidative damage by enhancing ERK1/2 phosphorylation [51]. Lopez et al. found that quercetin might be helpful in reducing oxidative damage of pancreatic insulinoma $\beta$ cells by regulating the polymerization tendency of human amylin, which is a pathological marker of T2DM [88].

5.1.6. Quercetin Inhibits Apoptosis. Modern investigations have shown that quercetin can reduce apoptosis in diabetic animals. By using the terminal dUTP nick end-labeling (TUNEL) method, Kanter et al. found that quercetin ameliorated testicular cell apoptosis of rats induced by diabetes [89]. Quercetin could significantly decrease the levels of proapoptotic markers (caspase-3, caspase-9, and Bax), while increasing the level of antiapoptotic markers (Bcl-2) in diabetic rats and mice $[75,87,90]$. Lin et al. also reported that quercetinrich guava juice lowered the expression of autophagy-related proteins (Beclin-1 and LC3-B) and a biomarker of pyroptosis (IL-1 $\beta$ ) in the pancreas and kidney of T2DM rats [90]. In addition, quercetin was reported to reduce pancreatic $\beta$ cells' apoptosis by increasing endothelial nitric oxide synthase 
(eNOS) and regulating the NO-cyclic $3^{\prime}, 5^{\prime}$-guanosine monophosphate (cGMP) signaling in endothelial cells [91].

Some studies have shown that quercetin can inhibit ER stress, which plays an important role in reducing apoptosis. Cai et al. reported that quercetin could inhibit ER stress to prevent glucosamine-induced RAW264.7 macrophages apoptosis [92]. Specifically, it decreased the expression of glucose regulated protein 78 (GRP78) and inhibited the activation of activating transcriptional factor $6 \alpha$ (ATF-6 $\alpha$ ) to reduce CCAAT/enhancer-binding protein (C/EBP) homologous protein (CHOP) expression and inhibit JNK phosphorylation and caspase-12 activation [92]. Another study found that quercetin treatment could also restore ER homeostasis by reducing the phosphorylation of protein kinase-like ER kinase (PERK) and the expression of caspase-3, thus reducing the apoptosis of human umbilical vein endothelial cells (HUVECs) [93].

5.2. Hyperlipidemia. Many investigations have shown that quercetin has good pharmacological activity on hyperlipidemia. It could reduce the levels of TG, TC, LDL, and VLDL, inhibit 3-hydroxy-3-methylglutaryl-CoA (HMGCoA) reductase activity, and increase HDL levels in hyperlipidemic animals $[14,56,94]$. Sikder et al. found that quercetin could ameliorate HFD-induced dyslipidemia in Swiss albino mice [95]. In detail, quercetin treatment reduced serum TC, TG, and LDL levels by $30 \%, 34 \%$, and $22 \%$, respectively. Cholesterol and TG levels were also reduced by $32 \%$ and $21 \%$, respectively, by quercetin treatment [95]. Similarly, Mariee et al. reported that quercetin administration for 3 weeks could significantly reduce the levels of serum TC (20\%), liver TC (22\%), liver TG (24\%), and serum LDL cholesterol (LDL-c) (31\%) and significantly increase serum HDL cholesterol (HDL-c) levels [96]. Juzwiak et al. studied the effect of quercetin on experimental hyperlipidemia in rabbits. The results demonstrated that quercetin taken for 12 weeks could effectively reduce serum TG and cholesterol levels elevated by high-fat diet [97]. A recent study indicated that pretreatment with quercetin by oral gavage for a period of 30 days decreased TC and increased HDL-c levels in hyperlipidemic rats induced by P-407 [98]. Moreover, quercetin could decrease plasma cholesterol and prevent the hypertrophy of the left ventricular in hypercholesterolemic mice [99]. Hu et al. also found that quercetin at 50 and 100 $\mathrm{mg} / \mathrm{kg}$ could effectively improve fructose-induced dyslipidemia in rats. It significantly lowered the serum TG, TC, and VLDL levels in rats [100].

The mechanism of quercetin's antihyperlipidemic effect may involve multiple aspects (Figure 2). Quercetin could reduce high blood cholesterol levels by specifically inhibiting the absorption of intestinal cholesterol through reducing the expression of the epithelial cholesterol transporter NiemannPick C1-like 1 (NPC1L1) [101]. Jung et al. and Kobori et al. found that quercetin supplementation improved dyslipidemia through reducing oxidative stress, increasing PPAR $\alpha$ expression, and improving the expression of some genes related to lipid metabolism in mice, including farnesyltransferase CAAX box $\alpha$ (Fnta), paraoxonase 1 (Pon1), aldehyde dehydrogenase 1 family member B1 (Aldh1b1), ATP- binding cassette subfamily G member 5 (Abcg5), apolipoprotein A-IV (Apoa4), acetyl-coenzyme A carboxylase $\alpha$ (Acaca), fatty acid synthase (FAS), cluster of differentiation 36 (CD36), glycerol-3-phosphate acyltransferase mitochondrial (Gpam), and sterol regulatory element-binding protein-1c (SREBP-1c) [102, 103]. Another study indicated that quercetin might treat hyperlipidemia by promoting PPAR $\gamma$ and liver X receptor $\alpha(\mathrm{LXR} \alpha)$ expressions to upregulate ATP-binding cassette transporter A1 (ABCA1) genes and increasing cholesterol efflux from THP-1 macrophages in human acute monocytic leukemia cells [104].

5.3. NAFLD. The pathogenesis of NAFLD involves multiple mechanisms, such as lipid accumulation, inflammation, and oxidative stress. Emerging evidence has shown that quercetin can effectively treat NAFLD by reducing lipid accumulation, antioxidation, and anti-inflammation and restoring disturbed metabolites and gut microbiota (Figure 2).

5.3.1. Quercetin Ameliorates Hepatic Lipid Accumulation. A high-fat and high-sucrose (HFS) diet can induce NAFLD in male rats. Compared with the HFS diet group, quercetin was found to significantly reduce the content of TC and TG in the liver of NAFLD rats [105]. Meanwhile, the assays for hepatic enzymes involved in lipid metabolism showed that the activity of glucose-6-phosphate dehydrogenase (G6PDH) was significantly increased, while the activity of hepatic lipase and glycerol-3-phosphate dehydrogenase (G3PDH) was significantly decreased by quercetin. Moreover, in HFD-induced nonalcoholic steatohepatitis (NASH) in rats, the concentrations of lipids and lipoproteins, including free fatty acids (FFAs), free cholesterol, esterified cholesterol, TC, TG, LDL, and VLDL, were significantly reduced after 4 weeks of treatment with quercetin $(20 \mathrm{mg} / \mathrm{kg})$ [106]. It was reported that quercetin could reduce hepatic lipid accumulation, thereby improving HFD-induced NAFLD by promoting hepatic VLDL assembly and lipophagy via the inositol-requiring transmembrane kinase/endoribonuclease $1 \alpha / \mathrm{X}$-box-binding protein 1 (IRE $1 \alpha / \mathrm{XBP} 1$ s) pathway. Specifically, compared with the HFD group, quercetin decreased hepatic TG content by $39 \%$ and TC by $28 \%$, resulted in a 1.5-fold increase in hepatic VLDL, and upregulated the expression of spliced XBP1s [107]. Pisonero-Vaquero et al. found that quercetin reduced lipid accumulation by modulating lipid metabolism-related gene expression via the PI3K/Akt pathway inactivation in a diet-induced mouse model of NAFLD [108]. Specifically, it significantly downregulated the expression of two de novo lipogenesis genes including SREBP-1C and LXR $\alpha$ and a fatty acid uptake- and trafficking-related gene, fatty acid translocase CD36 (FAT/CD36). Moreover, quercetin was able to upregulate the expression of fatty acid transport protein 5 (FATP5), fatty acid-binding protein 1 (FABP1), forkhead box protein A1 (FOXA1), and small heterodimer partner (SHP) [108]. Similarly, Li et al. proved that quercetin could significantly improve hepatic lipid accumulation and decrease the levels of TG by suppressing two lipogenesis gene expression levels of SREBP-1c and FAS [109]. Recently, Liu et al. found that quercetin could prevent FFA-induced lipid accumulation 
in vitro and alleviate $\mathrm{HFD}$-induced hepatic steatosis in vivo through increasing frataxin-mediated PTEN-induced putative kinase 1 (PINK1)/Parkin-dependent mitophagy [110]. In addition, HFD-induced NAFLD is usually accompanied by oxidized low-density lipoprotein (ox-LDL) deposition in the liver. Liu et al. found that quercetin administration for 24 weeks $(100 \mathrm{mg} / \mathrm{kg})$ could significantly alleviate HFDinduced liver damage and reduce hepatic cholesterol and ox-LDL levels by improving the autophagy lysosomal signaling pathway and inhibiting the expression of scavenger receptors, including macrophage scavenger receptor 1 (MSR1) and CD36 [111]. Recently, a study showed that quercetin could reduce liver lipid accumulation in T2DMinduced NAFLD by activating the farnesoid X receptor 1/ Takeda G protein-coupled receptor 5 (FXR1/TGR5) signaling pathway [112].

5.3.2. Quercetin Alleviates Inflammation and Oxidative Stress in Hepatitis Animals. NASH is the inflammatory subtype of NAFLD. A study reported that quercetin treatment $(20 \mathrm{mg} / \mathrm{kg})$ showed a protective effect against HFD-induced $\mathrm{NASH}$ by reducing the levels of inflammatory markers TNF- $\alpha$ and myeloperoxidase (MPO) [113]. Similarly, Ying et al. found that quercetin could reduce serum levels of proinflammatory cytokines TNF- $\alpha$ and IL- 6 by upregulating SIRT1 and downregulating iNOS and NF- $\kappa$ B p 65 in the NASH gerbils induced by a high-fat diet [114]. Moreover, Marcolin et al. reported that quercetin $(50 \mathrm{mg} / \mathrm{kg})$ could ameliorate inflammation and fibrosis in NASH mice by attenuating various proinflammatory and profibrotic gene pathways [115]. Specifically, it could significantly reduce the liver mRNA levels of IL-6, TNF- $\alpha$, high-mobility group box 1 (Hmgb1), and cyclooxygenase-2 (Ptgs2), and the liver protein concentration of Toll-like receptor-4 (TLR-4).

In addition, a study reported that quercetin exhibited significant antioxidant property in experimental NASH rats [116]. Nonenzymatic antioxidant glutathione (GSH) and several antioxidant enzymes including CAT, SOD, GSH-Px, glutathione reductase (GR), and glutathione-S-transferase (GST) were significantly increased by quercetin $(20 \mathrm{mg} / \mathrm{kg})$. Similarly, Surapaneni et al. reported that quercetin treatment could significantly reduce the level of prooxidant enzyme Cytochrome P450 2E1 (CYP2E1) in the liver of NASH rats, thereby improving CYP2E-mediated oxidative stress [117]. Another study found that quercetin could attenuate lipoperoxidation by regulating inflammatory and oxidative/nitrosative stress-related genes in a diet-induced mouse model of NAFLD [108]. Specifically, it effectively reduced the mRNA levels of TNF- $\alpha$, iNOS, osteopontin (OPN), and suppressor of cytokine signaling (SOCS) 3 .

5.3.3. Quercetin Regulates Serum Metabolites and Gut Microbiota in NAFLD Animals. Metabolomics is a common approach to study the pharmacological activity and molecular mechanism of natural products [118]. Recently, Xu et al. investigated the effects and mechanisms of quercetin on HFD-induced NAFLD using nontargeted metabolomics technology [119]. The results indicated that quercetin showed good hepatoprotective activity in 30-day NAFLD rats, and the underlying mechanisms might be related to its regulation of 2 inflammation-related metabolites (arachidonic acid and 12(S)-HPETE), 5 fatty acid-related metabolites (eicosapentaenoic acid, adrenic acid, oleic acid, docosahexaenoic acid, and palmitic acid), 1 oxidative stress-related metabolite (2-hydroxybutyric acid), and 5 other differential metabolites ( $\alpha$-dimorphecolic acid, citric acid, 15(S)-hydroxyeicosatrienoic acid, chenodeoxycholic acid glycine conjugate, and 9,10,13-trihydroxy-11-octadecenoic acid) [119].

In recent years, many studies have shown that gut microbiota is involved in the progression of NAFLD [120-122]. Porras et al. found that the protective effect of quercetin on NAFLD is also mediated by modulating gut microbiota disorders and related gut-liver axis [123]. Specifically, supplementation of quercetin for 16 weeks could significantly reduce the Firmicutes/Bacteroidetes ratio and the relative percentage of Proteobacteria phylum, and increase the total bacteria concentration in HFD-induced NAFLD mice. At the genus level, quercetin was found to considerably reduce the number of reads of Desulfovibrio and Helicobacter, and significantly increase the relative abundance of Flavobacterium, Allobaculum, and Sutterella. Moreover, this study also proved that quercetin could increase the concentration of beneficial metabolites derived from gut microbiota (acetate, propionate, and butyrate), restore the gut barrier function, and improve endotoxemia, gut-liver axis activation, and subsequent inflammatory gene overexpression in NAFLD mice [123]. Subsequently, they further studied the protective role of quercetin against NAFLD by gut microbiota transplantation. The results showed that gut microbiota transplantation from the HFD-nonresponder donor and the HFD-fed donor with the highest response to quercetin resulted in a protective phenotype against the development of NAFLD [124]. Recently, they revealed that this protective phenotype against NAFLD was associated with the increase of gut Desulfovibrio and Oscillospira, the decrease of gut Bacteroides and Oribacterium, the increase of secondary bile acids (BAs) in plasma and feces, the induction of hepatic BA transporters, and the inhibition of hepatic lipogenic and BA synthesis genes [125]. The above findings suggest that quercetin has good prebiotic capacity, and regulation of gut microbiota is one of the important mechanisms of quercetin in the treatment of NAFLD.

\section{Clinical Evidence of Quercetin in Treating the Three Metabolic Diseases}

Although there is a lot of in vitro and in vivo evidence that quercetin could be a promising therapeutic agent for diabetes, hyperlipidemia, and NAFLD, clinical trials must confirm its effectiveness in humans. Table 2 summarizes the published clinical trials of quercetin in the treatment of the three diseases.

6.1. Diabetes. Several cohort studies have estimated the association between daily quercetin intake and the onset of diabetes. Yao et al. conducted a large-scale cross-sectional study on 14711 Chinese adults [13]. It was found that the daily intake of quercetin in Chinese adults was $20.9 \pm 2.32 \mathrm{mg}$, and the 


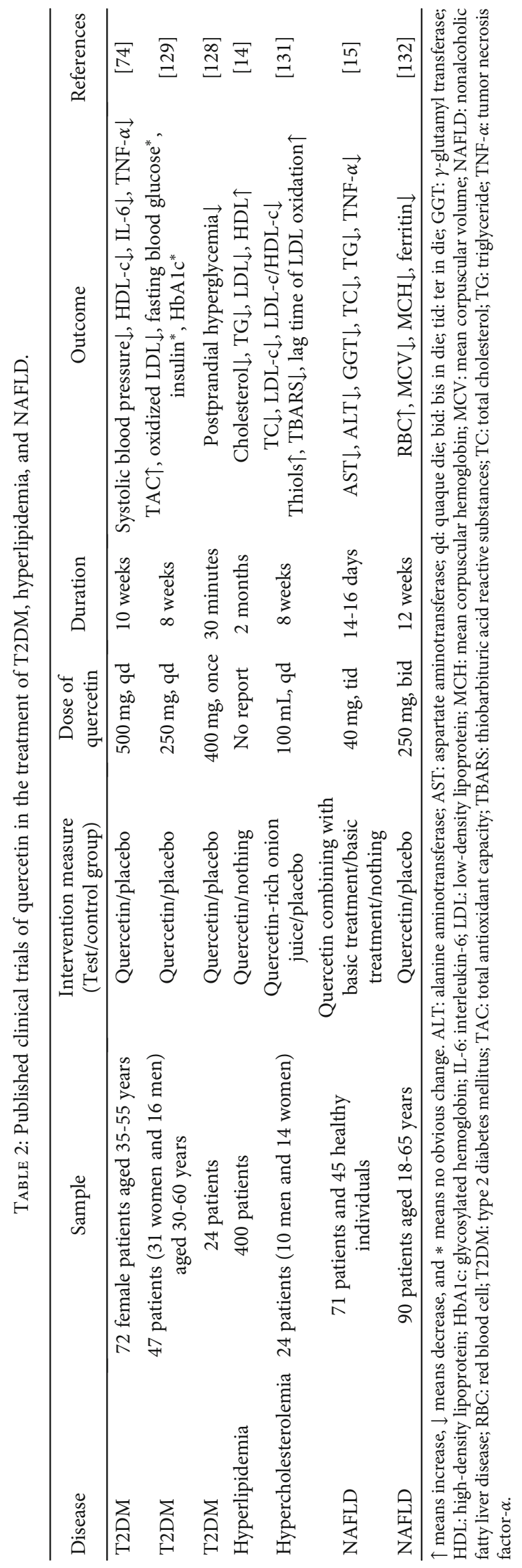


intake of quercetin was significantly negatively correlated with the prevalence of T2DM. Another prospective study conducted a survey on 10054 Finnish citizens [126]. The daily intake of quercetin was $3.3 \pm 2.4 \mathrm{mg}$, and a total of 526 cases of T2DM occurred during the follow-up period. More importantly, they found that a lower risk of T2DM tended to be associated with higher intake of quercetin and myricetin. Moreover, a randomized, double-blind, placebocontrolled crossover trial of 37 apparently healthy, nonsmoking adults found that supplementation with quercetin could significantly reduce the plasma concentration of methylglyoxal, a compound associated with diabetes and its complications [127]. These results indicate that quercetin may have beneficial effects in improving diabetes.

Currently, a small number of clinical trials have been implemented to study the impacts of quercetin on human subjects with diabetes and related disorders. A randomized blinded crossover study was designed to evaluate the beneficial effect of quercetin on postprandial hyperglycemia challenged with carbohydrates load in T2DM patients. The results showed that a single oral dose of quercetin $(400 \mathrm{mg}$ ) could effectively suppress postprandial hyperglycemia in T2DM patients after maltose loading [128]. Zahedi et al. conducted a 10-week double-blind randomized clinical trial to evaluate the effects of quercetin on blood lipids, blood pressure, and inflammatory biomarkers in patients with T2DM [74]. 72 women with T2DM were divided into quercetin group and placebo group. Quercetin was given to participants at a dose of $500 \mathrm{mg}$ per day. Compared with placebo, quercetin administration significantly reduced the patient's systolic blood pressure. Moreover, quercetin could significantly reduce serum HDL-c, TNF- $\alpha$, and IL- 6 levels, but there is no significant difference compared with the placebo group. In addition, an 8-week randomized clinical trial was conducted to study the effects of quercetin on glycemic control, oxidative stress, lipid profile, and insulin resistance in T2DM [129]. The results showed that oral quercetin $(250 \mathrm{mg} /$ day) for 8 weeks could significantly improve the antioxidant status of patients with T2DM. However, it did not significantly change the fasting blood glucose, serum insulin, and glycosylated hemoglobin (HbAlc) levels, and lipid profile in patients with T2DM. The difference between the results of these clinical trials may be due to the different doses of quercetin utilized and the duration of intervention. Recently, a systematic review and meta-analysis was performed to determine the effect of quercetin on glycemic control among patients with metabolic disorders [130]. This study concluded that quercetin can significantly reduce fasting plasma glucose levels when the dose is $\geq 500 \mathrm{mg} /$ day and the duration is $\geq 8$ weeks. Therefore, the dose and duration of intervention are the key factors that determine whether quercetin can effectively treat diabetes.

6.2. Hyperlipidemia. A double-blind randomized clinical trial was conducted to determine the effects of regular consumption of quercetin on blood lipid levels among asymptomatic persons with dyslipidemia. The results showed that giving quercetin for two months could decrease the levels of cholesterol, TG, and LDL, and increase HDL levels [14]. Moreover,
Lu et al. studied the hypocholesterolemic effect of quercetinrich onion juice on healthy mild hypercholesterolemia [131]. 24 subjects were divided into two groups, and they consumed $100 \mathrm{~mL}$ of onion juice or placebo every day. After 8 weeks of intervention, quercetin-rich onion juice significantly decreased waist circumference, TC, LDL-c, and LDL-c/ HDL-c levels, elevated total antioxidation capacity, and prolonged the lag time of LDL oxidation. Although these two studies indicate that quercetin may have a positive effect on blood lipid levels, a meta-analysis of randomized controlled trials has yielded mixed results. The meta-analysis concluded that quercetin does not have any clinically relevant effects on plasma lipids, except for a significant reduction in TG at doses above $50 \mathrm{mg} /$ day [9]. Moreover, the impact of quercetin on TG levels was found to be significantly associated with its dosage and duration of supplementation. Therefore, due to the small number of clinical trials and the limited sample size, the effectiveness of quercetin on hyperlipidemia cannot be reached with a definite conclusion, although certain trends can be observed. More clinical studies with larger sample sizes and longer trial durations are needed to verify its effectiveness against hyperlipidemia in human subjects.

6.3. NAFLD. So far, there are few clinical studies on the therapeutic effect of quercetin on NAFLD. Prysyazhnyuk and Voloshyn studied the effects of quercetin on blood biochemical indicators and pro- and anti-inflammatory cytokines in patients with NAFLD [15]. After 2 weeks of quercetin combined with basic treatment, the activities of aspartate aminotransferase (AST), alanine aminotransferase (ALT), and gamma-glutamyl transferase (GGT) were significantly reduced by $37.2 \%, 50.4 \%$, and $89.9 \%$, respectively. Moreover, the levels of TC, TG, and TNF- $\alpha$ significantly decreased by $16.7 \%, 33.3 \%$, and $39.8 \%$, respectively. These results suggest that quercetin has potential therapeutic value for NAFLD. Oxidative stress plays a key role in the pathogenesis of NAFLD and can also mediate the destruction and death of red blood cell (RBC). Recently, a randomized, double-blind, placebo-controlled trial was conducted to evaluate the effect of quercetin supplementation on hematological parameters in patients with NAFLD [132]. Specifically, 90 patients were supplemented with quercetin or placebo capsules twice a day for 12 weeks. Compared with the placebo group, the quercetin treatment group significantly increased RBC levels, but decreased the mean corpuscular volume, mean corpuscular hemoglobin, and ferritin levels.

As noted, the current clinical trials show that quercetin has a beneficial effect on some biomarkers associated with NAFLD. However, clinical studies on the effects of quercetin in NAFLD remain scarce. The number of patients enrolled in these trials is also small. Therefore, more high-quality clinical trials are needed to better understand the efficacy of quercetin on NAFLD in a wide range of populations.

\section{Conclusions and Future Perspectives}

Quercetin is a widely distributed natural flavonol compound. It can be obtained not only from daily vegetables and fruits (e.g., onions and apples) but also from some common 
medicinal plants (e.g., G. biloba and $P$. orientale). In recent years, quercetin has received widespread attention due to its exciting pharmacological potential and health benefits. In this review, we comprehensively summarized the pharmacological effects and molecular mechanisms of quercetin in the treatment of three metabolic diseases (diabetes, hyperlipidemia, and NAFLD). The information can provide useful references for future preclinical and clinical studies of quercetin.

Previous studies have shown that the oral bioavailability of quercetin is low, which will limit its therapeutic application. Therefore, an important issue that needs to be resolved in future research is to improve the bioavailability of quercetin. In recent years, nanotechnology has developed rapidly. Nanoformulations provide a promising strategy to solve the problems related to the bioavailability of quercetin [133]. Singh et al. found that quercetin-loaded nanomicelles could improve its bioavailability and antidiabetic activity [134]. Moreover, novel delivery forms (e.g., Quercetin Phytosome ${ }^{\circledR}$ ) can also enhance the bioavailability of quercetin. Riva et al. reported that Quercetin Phytosome ${ }^{\circledR}$ did not affect the metabolic control of diabetic patients treated with metformin, which supports the application of quercetin in diabetic patients with no effect on conventional metformin treatment [135]. Besides, as mentioned previously, quercetin metabolites are more easily absorbed than quercetin. Therefore, additional research is needed to determine whether the health benefits of quercetin are directly or indirectly mediated by its metabolites.

Accumulating evidences show that quercetin has beneficial effects on these three metabolic diseases through various mechanisms. It can improve diabetes by promoting insulin secretion, improving insulin resistance, regulating glucose homeostasis, and inhibiting inflammation, oxidative stress, and apoptosis. The therapeutic mechanisms of quercetin on hyperlipidemia also involve multiple aspects. It can inhibit the absorption of intestinal cholesterol, reduce oxidative stress, downregulate the expression of some adipogenic genes (e.g., FAS and SREBP-1c), and promote the expression of $\operatorname{PPAR} \alpha, \operatorname{PPAR} \gamma$, and $\operatorname{LXR} \alpha$. In addition, the therapeutic effect of quercetin on NAFLD can be attributed to its ability to ameliorate liver lipid accumulation, alleviate inflammation and oxidative stress, and regulate serum metabolic disorders.

In recent years, monotargeted drugs have been found to have limitations in the overall clinical outcome of patients, because diseases are usually caused by disturbances in multiple signaling pathways [136]. Moreover, monotargeted drugs are prone to drug resistance and side effects. Therefore, new drugs may need to undergo a shift from single target therapy to multitarget therapy. Multitargeted drugs may be less potent, but they can act on multiple targets to improve various symptoms of the disease [136]. As shown in Figure 2, quercetin can regulate a variety of signaling pathways, so it has multitarget therapeutic effects on the three metabolic diseases. However, some targets have only been proven at the cellular level or in an animal experiment. Therefore, more experiments are needed to confirm these targets. In future research, it is also necessary to identify the key target of quercetin in the treatment of these metabolic diseases by using gene knockout or gene silencing technology.
Many studies have shown that gut microbiota may be a potential therapeutic target for metabolic diseases [137]. Gut microbiota plays an important role in the treatment of diseases with herbal medicine through complex interactions [138]. Herbal chemicals closely affect gut microbiota composition. Reciprocally, the gut microbiota can biologically convert herbal chemicals into active metabolites. Therefore, regulating the gut microbiota or being metabolized by the gut microbiota may be the common ground for the therapeutic effect of quercetin on diabetes, hyperlipidemia, and NAFLD. As mentioned earlier, the regulation of gut microbiota has been proven to be one of the important mechanisms of quercetin in the treatment of NAFLD [123-125]. However, there are scarce studies on the role of gut microbiota in the treatment of diabetes and hyperlipidemia with quercetin. Only one study reported that quercetin improved STZinduced diabetic peripheral neuropathy in rats by decreasing four pathogenic species and enriching two prebiotic species [139]. Therefore, the relationship between the effects of quercetin on these metabolic diseases and the gut microbiota remains to be elucidated. Moreover, the identification of specific bacterial strains that may contribute to the therapeutic effect of quercetin on NAFLD still needs further research.

Up to now, clinical studies on the effects of quercetin in diabetes, hyperlipidemia, and NAFLD remain scarce. The beneficial effects of quercetin on these three diseases have been observed in some human clinical trials. However, some studies have also reported inconsistent or conflicting results, especially for diabetes. The dosage and duration of treatment may be the key factors affecting the clinical effectiveness of quercetin. Therefore, definitive conclusions cannot be drawn until more clinical data are obtained. Overall, although many animal studies have reported the therapeutic effects of quercetin on the three metabolic diseases, its clinical application in humans still has a long way to go. More clinical trials with appropriate design are needed to verify the true impact of quercetin treatment on patients with diabetes, hyperlipidemia, and NAFLD. Besides, additional researches are necessary to further evaluate the dose-response effect of quercetin on these three diseases. The completion of these studies is expected to provide a scientific basis for the therapeutic potential of quercetin in human subjects. As we gain a deeper understanding of the health benefits of quercetin, its therapeutic value for metabolic diseases may become more apparent and widely accepted.

\section{Data Availability}

No data were used to support this study.

\section{Conflicts of Interest}

The authors declare that there is no conflict of interest regarding the publication of this article.

\section{Authors' Contributions}

Huan Yi and Hengyang Peng contributed equally to this work. 


\section{Acknowledgments}

The authors gratefully acknowledge the financial support from the National Key Research and Development Program of China (No. 2019YFC1712302), the Major Cultivation Project of Scientific and Technological Achievements Transformation of Sichuan Provincial Department of Education (No. 18CZ0011), and the Open Research Fund of Chengdu University of Traditional Chinese Medicine Key Laboratory of Systematic Research of Distinctive Chinese Medicine Resources in Southwest China (2020JCRC007).

\section{References}

[1] K. R. Costello and D. E. Schones, "Chromatin modifications in metabolic disease: potential mediators of long-term disease risk," Wiley Interdisciplinary Reviews: Systems Biology and Medicine, vol. 10, no. 4, article e1416, 2018.

[2] P. Saeedi, I. Petersohn, P. Salpea et al., "Global and regional diabetes prevalence estimates for 2019 and projections for 2030 and 2045: results from the International Diabetes Federation Diabetes Atlas, 9(th) edition," Diabetes Research and Clinical Practice, vol. 157, 2019.

[3] Group DPPR, "10-year follow-up of diabetes incidence and weight loss in the diabetes prevention program outcomes study," The Lancet, vol. 374, pp. 1677-1686, 2009.

[4] L. K. Liu, F. P. Chou, Y. C. Chen, C. C. Chyau, H. H. Ho, and C. J. Wang, "Effects of mulberry (Morus alba L.) extracts on lipid homeostasis in vitro and in vivo," Journal of Agricultural and Food Chemistry, vol. 57, no. 16, pp. 7605-7611, 2009.

[5] R. S. Scott, C. J. Lintott, and M. J. Wilson, "Simvastatin and side effects," The New Zealand Medical Journal, vol. 104, no. 924, pp. 493-495, 1991.

[6] P. Kanwar and K. V. Kowdley, "The metabolic syndrome and its influence on nonalcoholic steatohepatitis," Clinics in Liver Disease, vol. 20, no. 2, pp. 225-243, 2016.

[7] R. Ali and K. Cusi, "New diagnostic and treatment approaches in non-alcoholic fatty liver disease (NAFLD)," Annals of Medicine, vol. 41, no. 4, pp. 265-278, 2009.

[8] M. Bacanli, S. A. Dilsiz, N. Basaran, and A. A. Basaran, "Effects of phytochemicals against diabetes," Advances in Food and Nutrition Research, vol. 89, pp. 209-238, 2019.

[9] A. Sahebkar, "Effects of quercetin supplementation on lipid profile: a systematic review and meta-analysis of randomized controlled trials," Critical Reviews in Food Science and Nutrition, vol. 57, no. 4, pp. 666-676, 2017.

[10] A. Maalik, F. A. Khan, A. Mumtaz et al., "Pharmacological applications of quercetin and its derivatives: a short review," Tropical Journal of Pharmaceutical Research, vol. 13, no. 9, pp. 1561-1566, 2014.

[11] X.-W. Zhang, J.-Y. Chen, D. Ouyang, and J.-H. Lu, "Quercetin in animal models of Alzheimer's disease: a systematic review of preclinical studies," International Journal of Molecular Sciences, vol. 21, no. 2, p. 493, 2020.

[12] W. Zhang, Y. Wang, Z. Yang et al., "Antioxidant treatment with quercetin ameliorates erectile dysfunction in streptozotocininduced diabetic rats," Journal of Bioscience and Bioengineering, vol. 112, no. 3, pp. 215-218, 2011.

[13] Z. Yao, Y. Gu, Q. Zhang et al., "Estimated daily quercetin intake and association with the prevalence of type 2 diabetes mellitus in Chinese adults," European Journal of Nutrition, vol. 58, no. 2, pp. 819-830, 2019.

[14] E. Talirevic and J. Sehovic, "Quercetin in the treatment of dyslipidemia," Medicinski Arhiv, vol. 66, no. 2, pp. 87-88, 2012.

[15] V. Prysyazhnyuk and O. Voloshyn, "Effects of comprehensive treatment with quercetin administration on biochemical blood parameters and pro-and anti-inflammatory cytokines in nonalcoholic fatty liver disease patients," The Pharma Innovation Journal, vol. 6, pp. 386-389, 2017.

[16] L. Xiao, G. Luo, Y. Tang, and P. Yao, "Quercetin and iron metabolism: what we know and what we need to know," Food and Chemical Toxicology, vol. 114, pp. 190-203, 2018.

[17] H. Nishimuro, H. Ohnishi, M. Sato et al., "Estimated daily intake and seasonal food sources of quercetin in Japan," Nutrients, vol. 7, no. 4, pp. 2345-2358, 2015.

[18] S. M. Somerset and L. Johannot, "Dietary flavonoid sources in Australian adults," Nutrition and Cancer, vol. 60, no. 4, pp. 442-449, 2008.

[19] S. H. Hakkinen, S. O. Karenlampi, I. M. Heinonen, H. M. Mykkanen, and A. R. Torronen, "Content of the flavonols quercetin, myricetin, and kaempferol in 25 edible berries," Journal of Agricultural and Food Chemistry, vol. 47, no. 6, pp. 2274-2279, 1999.

[20] N. Verma and N. Trehan, "HPLC analysis of methanolic extract of herbs for quercetin content," Journal of Pharmacognosy and Phytochemistry, vol. 2, pp. 159-162, 2013.

[21] B. S. Patil, L. M. Pike, and K. S. Yoo, "Variation in the quercetin content in different colored onions (Allium cepa L.)," Journal of the American Society for Horticultural Science, vol. 120, no. 6, pp. 909-913, 1995.

[22] H. G. Ulusoy and N. Sanlier, "A minireview of quercetin: from its metabolism to possible mechanisms of its biological activities," Critical Reviews in Food Science and Nutrition, vol. 60, no. 19, pp. 3290-3303, 2020.

[23] P. Mukhopadhyay and A. K. Prajapati, "Quercetin in antidiabetic research and strategies for improved quercetin bioavailability using polymer-based carriers-a review," RSC Advances, vol. 5, no. 118, pp. 97547-97562, 2015.

[24] X. M. Yin, L. Y. Chen, and X. W. Zhang, "Determination of the quercetin content in the fructus polygoni orientalis," Journal of Medical Science Yanbian University, vol. 4, pp. 264-265, 2005.

[25] D. W. Wang and K. F. Ma, "Content determination of quercetin in Crataegus sanguinea by HPLC," Shizhen Guo Yi Guo Yao, vol. 19, pp. 2214-2215, 2008.

[26] Y. C. Quan, L. P. Guan, and G. H. Zheng, "Study on determination of quercetin in hawthorn leaf by HPLC assay," Shizhen Guo Yi Guo Yao, vol. 17, pp. 207-208, 2006.

[27] S. Q. Wu and D. W. Chen, "Determination and significance of quercetin in Huaimi by HPLC," The Journal of Medical Theory and Practice, vol. 18, pp. 730-731, 2005.

[28] X. Zhou, Z. M. Wang, J. Zheng, L. W. Zhao, and Z. Y. Liu, "Determination of quercetin in Chinese herbal medicine by capillary electrophoresis," Chemical Research in Chinese Universities, vol. 26, pp. 657-659, 2005.

[29] Y. D. Deng, Z. H. Yan, and X. D. Zhou, "Determination of quercetin in guava leaf, Momordica charantiap, Kuding tea by high-performance liquid chromatography," Modern Medicine Journal of China, vol. 13, pp. 31-32, 2011. 
[30] D. Z. Yang and X. P. Qi, "HPLC for content measurement of quercetin in Polygonum perfoliatum," Asia-Pacific Traditional Medicine, vol. 7, pp. 26-27, 2011.

[31] Y. Wei, X. J. Ding, and J. Y. Bao, "Determination of quercetin in Yuganzi by HPLC," Shizhen Guo Yi Guo Yao, vol. 19, pp. 1634-1635, 2008.

[32] M. Y. Liu, G. P. Xue, C. S. Zhang, L. X. Shen, and J. F. Wang, "Determination of quercetin in Cuscuta chinensis by HPLC," Shizhen Guo Yi Guo Yao, vol. 23, pp. 269-270, 2012.

[33] X. Lin, B. M. Liu, and M. S. Chen, "The determination of quercetin in Baeckea frutescens L. by HPLC," Shizhen Guo Yi Guo Yao, vol. 20, pp. 102-103, 2009.

[34] L. Lv, Y. M. Zhu, and D. M. Xu, "Study on flavones from Taxillus chinensis (DC.) Danser and assay of its quercetin," Chinese Traditional Patent Medicine, vol. 26, pp. 10461048, 2004.

[35] W. S. Ren, T. Y. Bai, and F. H. Chang, "Determination of quercetin in Clematis brevicaudata DC by HPLC," Zhongguo Min Zu Yi Yao Za Zhi, vol. 16, pp. 50-51, 2010.

[36] M. M. Zhou and L. Ge, "Determination of quercetin content in Dracoephalum mololariea L. by RP-HPLC," Chinese Journal of Ethnomedicine and Ethnopharmacy, vol. 19, pp. 1-2, 2010.

[37] H. P. Liu, X. F. Shi, Y. C. Zhang, Z. X. Li, L. Zhang, and Z. Y. Wang, "Quantitative analysis of quercetin in Euphorbia helioscopia L by RP-HPLC," Cell Biochemistry and Biophysics, vol. 61, no. 1, pp. 59-64, 2011.

[38] T. Mamat and X. L. Ding, "RP-HPLC determination of quercetin in Brassica rapa L. for Uighur medicine," Chinese Journal of Pharmaceutical Analysis, vol. 31, pp. 1420-1422, 2011.

[39] C. G. M. Heijnen, G. R. M. M. Haenen, R. Minou Oostveen, E. M. Stalpers, and A. Bast, "Protection of flavonoids against lipid peroxidation: the structure activity relationship revisited," Free Radical Research Communications, vol. 36, no. 5, pp. 575-581, 2002.

[40] W. Wang, C. Sun, L. Mao et al., "The biological activities, chemical stability, metabolism and delivery systems of quercetin: a review," Trends in Food Science and Technology, vol. 56, pp. 21-38, 2016.

[41] Y. Guo and R. S. Bruno, "Endogenous and exogenous mediators of quercetin bioavailability," The Journal of Nutritional Biochemistry, vol. 26, no. 3, pp. 201-210, 2015.

[42] X. Chen, O. Q. Yin, Z. Zuo, and M. S. Chow, "Pharmacokinetics and modeling of quercetin and metabolites," Pharmaceutical Research, vol. 22, no. 6, pp. 892-901, 2005.

[43] Y. Li, J. Yao, C. Han et al., "Quercetin, inflammation and immunity," Nutrients, vol. 8, no. 3, 2016.

[44] S. V. Luca, I. Macovei, A. Bujor et al., "Bioactivity of dietary polyphenols: the role of metabolites," Critical Reviews in Food Science and Nutrition, vol. 60, no. 4, pp. 626-659, 2020.

[45] K. Murota and J. Terao, "Quercetin appears in the lymph of unanesthetized rats as its phase II metabolites after administered into the stomach," FEBS Letters, vol. 579, no. 24, pp. 5343-5346, 2005.

[46] I. Erlund, "Review of the flavonoids quercetin, hesperetin, and naringenin. Dietary sources, bioactivities, bioavailability, and epidemiology," Nutrition Research, vol. 24, no. 10, pp. 851-874, 2004.

[47] L. Rangel-Ordóñez, M. Nöldner, M. Schubert-Zsilavecz, and M. Wurglics, "Plasma levels and distribution of flavonoids in rat brain after single and repeated doses of standardized
Ginkgo biloba extract EGb 761 ${ }^{\circledR}, "$ Planta Medica, vol. 76, no. 15, pp. 1683-1690, 2010.

[48] S. Bhattacharya, N. Oksbjerg, J. F. Young, and P. B. Jeppesen, "Caffeic acid, naringenin and quercetin enhance glucosestimulated insulin secretion and glucose sensitivity in INS1E cells," Diabetes, Obesity \& Metabolism, vol. 16, no. 7, pp. 602-612, 2014.

[49] C. Carrasco-Pozo, K. N. Tan, M. Reyes-Farias et al., "The deleterious effect of cholesterol and protection by quercetin on mitochondrial bioenergetics of pancreatic $\beta$-cells, glycemic control and inflammation: In vitro and in vivo studies," Redox Biology, vol. 9, pp. 229-243, 2016.

[50] N. Suganya, S. Dornadula, S. Chatterjee, and R. K. Mohanram, "Quercetin improves endothelial function in diabetic rats through inhibition of endoplasmic reticulum stressmediated oxidative stress," European Journal of Pharmacology, vol. 819, pp. 80-88, 2018.

[51] E. Youl, G. Bardy, R. Magous et al., "Quercetin potentiates insulin secretion and protects INS-1 pancreatic $\beta$-cells against oxidative damage via the ERK1/2 pathway," British Journal of Pharmacology, vol. 161, no. 4, pp. 799-814, 2010.

[52] G. Bardy, A. Virsolvy, J. F. Quignard et al., "Quercetin induces insulin secretion by direct activation of L-type calcium channels in pancreatic beta cells," British Journal of Pharmacology, vol. 169, no. 5, pp. 1102-1113, 2013.

[53] M. Zhuang, H. Qiu, P. Li, L. Hu, Y. Wang, and L. Rao, "Islet protection and amelioration of type 2 diabetes mellitus by treatment with quercetin from the flowers of Edgeworthia gardneri," Drug Design, Development and Therapy, vol. 12, pp. 955-966, 2018.

[54] M. Kittl, M. Beyreis, M. Tumurkhuu et al., "Quercetin stimulates insulin secretion and reduces the viability of rat INS-1 beta-cells," Cellular Physiology and Biochemistry, vol. 39, no. 1, pp. 278-293, 2016.

[55] A. E. A. El-Baky, "Quercetin protective action on oxidative stress, sorbitol, insulin risistance and $\beta$-cells function in expermintal diabetic rats," International Journal of Pharmaceutical Studies and Research, vol. 2, pp. 11-18, 2011.

[56] Y. Shao, Y. Yu, C. Li, J. Yu, R. Zong, and C. Pei, "Synergistic effect of quercetin and 6-gingerol treatment in streptozotocin induced type 2 diabetic rats and poloxamer P-407 induced hyperlipidemia," RSC Advances, vol. 6, no. 15, pp. 1223512242, 2016.

[57] H. H. Gaballah, S. S. Zakaria, S. E. Mwafy, N. M. Tahoon, and A. M. Ebeid, "Mechanistic insights into the effects of quercetin and/or GLP-1 analogue liraglutide on high-fat diet/streptozotocin-induced type 2 diabetes in rats," Biomedicine \& Pharmacotherapy, vol. 92, pp. 331-339, 2017.

[58] C. C. Chuang, K. Martinez, G. Xie et al., "Quercetin is equally or more effective than resveratrol in attenuating tumor necrosis factor-\{alpha\}-mediated inflammation and insulin resistance in primary human adipocytes," The American Journal of Clinical Nutrition, vol. 92, no. 6, pp. 1511-1521, 2010.

[59] Q. Y. Zhang, Y. Pan, R. Wang et al., "Quercetin inhibits AMPK/TXNIP activation and reduces inflammatory lesions to improve insulin signaling defect in the hypothalamus of high fructose-fed rats," The Journal of Nutritional Biochemistry, vol. 25, no. 4, pp. 420-428, 2014.

[60] R. Dhanya, A. D. Arya, P. Nisha, and P. Jayamurthy, "Quercetin, a lead compound against type 2 diabetes ameliorates 
glucose uptake via AMPK pathway in skeletal muscle cell line," Frontiers in Pharmacology, vol. 8, p. 336, 2017.

[61] M. S. Sandeep and C. D. Nandini, "Influence of quercetin, naringenin and berberine on glucose transporters and insulin signalling molecules in brain of streptozotocin-induced diabetic rats," Biomedicine \& Pharmacotherapy, vol. 94, pp. 605-611, 2017.

[62] H. M. Eid, A. Nachar, F. Thong, G. Sweeney, and P. S. Haddad, "The molecular basis of the antidiabetic action of quercetin in cultured skeletal muscle cells and hepatocytes," Pharmacognosy Magazine, vol. 11, no. 41, pp. 74-81, 2015.

[63] D. K. Yang and H. S. Kang, "Anti-diabetic effect of cotreatment with quercetin and resveratrol in streptozotocininduced diabetic rats," Biomolecules \& Therapeutics, vol. 26, no. 2, pp. 130-138, 2018.

[64] J. Peng, Q. Li, K. Li et al., "Quercetin improves glucose and lipid metabolism of diabetic rats: involvement of Akt signaling and SIRT1," Journal Diabetes Research, vol. 2017, article 3417306, 10 pages, 2017.

[65] M. M. Alam, D. Meerza, and I. Naseem, "Protective effect of quercetin on hyperglycemia, oxidative stress and DNA damage in alloxan induced type 2 diabetic mice," Life Sciences, vol. 109, no. 1, pp. 8-14, 2014.

[66] H. M. Eid, L. C. Martineau, A. Saleem et al., "Stimulation of AMP-activated protein kinase and enhancement of basal glucose uptake in muscle cells by quercetin and quercetin glycosides, active principles of the antidiabetic medicinal plant Vaccinium vitis-idaea," Molecular Nutrition \& Food Research, vol. 54, no. 7, pp. 991-1003, 2010.

[67] N. Xiao, F. Mei, Y. Sun, G. Pan, B. Liu, and K. Liu, "Quercetin, luteolin, and epigallocatechin gallate promote glucose disposal in adipocytes with regulation of AMP-activated kinase and/or sirtuin 1 activity," Planta Medica, vol. 80, no. 12, pp. 993-1000, 2014.

[68] M. Vessal, M. Hemmati, and M. Vasei, "Antidiabetic effects of quercetin in streptozocin-induced diabetic rats," Comparative Biochemistry and Physiology Part C: Toxicology \& Pharmacology, vol. 135, no. 3, pp. 357-364, 2003.

[69] G. Oboh, A. O. Ademosun, P. O. Ayeni, O. S. Omojokun, and F. Bello, "Comparative effect of quercetin and rutin on $\alpha$ amylase, $\alpha$-glucosidase, and some pro-oxidant-induced lipid peroxidation in rat pancreas," Comparative Clinical Pathology, vol. 24, pp. 1103-1110, 2014.

[70] D. Fontana Pereira, L. H. Cazarolli, C. Lavado et al., "Effects of flavonoids on $\alpha$-glucosidase activity: potential targets for glucose homeostasis," Nutrition, vol. 27, no. 11-12, pp. 1161-1167, 2011.

[71] R. A. Rifaai, N. F. El-Tahawy, and S. E. Ali, "Effect of quercetin on the endocrine pancreas of the experimentally induced diabetes in male albino rats: a histological and immunohistochemical study," Journal of Diabetes \& Metabolism, vol. 3, p. 3, 2012.

[72] H. E. Eitah, Y. A. Maklad, N. F. Abdelkader, A. A. Gamal El Din, M. A. Badawi, and S. A. Kenawy, "Modulating impacts of quercetin/sitagliptin combination on streptozotocininduced diabetes mellitus in rats," Toxicology and Applied Pharmacology, vol. 365, pp. 30-40, 2019.

[73] L. Rivera, R. Moron, M. Sanchez, A. Zarzuelo, and M. Galisteo, "Quercetin ameliorates metabolic syndrome and improves the inflammatory status in obese Zucker rats," Obesity (Silver Spring), vol. 16, no. 9, pp. 2081-2087, 2008.
[74] M. Zahedi, R. Ghiasvand, A. Feizi, G. Asgari, and L. Darvish, "Does quercetin improve cardiovascular risk factors and inflammatory biomarkers in women with type 2 diabetes: a double-blind randomized controlled clinical trial," International Journal of Preventive Medicine, vol. 4, no. 7, pp. 777785, 2013.

[75] J. Roslan, N. Giribabu, K. Karim, and N. Salleh, "Quercetin ameliorates oxidative stress, inflammation and apoptosis in the heart of streptozotocin-nicotinamide-induced adult male diabetic rats," Biomedicine \& Pharmacotherapy, vol. 86, pp. 570-582, 2017.

[76] N. F. Fedosova, S. V. Alisievich, K. V. Lyadov, E. P. Romanova, I. A. Rud'ko, and A. A. Kubatiev, "Mechanisms underlying diquertin-mediated regulation of neutrophil function in patients with non-insulin-dependent diabetes mellitus," Bulletin of Experimental Biology and Medicine, vol. 137, no. 2, pp. 143-146, 2004.

[77] O. Salah and M. D. Bashir, "Hepatoprotective role for quercetin in diabetic rats: hypolipidemic and antioxidant effects," The Medical Journal of Cairo University, vol. 82, pp. 169178, 2014.

[78] S. M. Jeong, M. J. Kang, H. N. Choi, J. H. Kim, and J. I. Kim, "Quercetin ameliorates hyperglycemia and dyslipidemia and improves antioxidant status in type 2 diabetic $\mathrm{db} / \mathrm{db}$ mice," Nutrition Research and Practice, vol. 6, no. 3, pp. 201-207, 2012.

[79] H. Elbe, M. Esrefoglu, N. Vardi, E. Taslidere, E. Ozerol, and K. Tanbek, "Melatonin, quercetin and resveratrol attenuates oxidative hepatocellular injury in streptozotocin-induced diabetic rats," Human \& Experimental Toxicology, vol. 34, no. 9, pp. 859-868, 2015.

[80] A. Senyigit, S. Durmus, E. B. Mirzatas et al., "Effects of quercetin on lipid and protein damage in the liver of streptozotocin-induced experimental diabetic rats," Journal of Medicinal Food, vol. 22, no. 1, pp. 52-56, 2019.

[81] S. O. Adewole, E. A. Caxton-Martins, and J. A. O. Ojewole, "Protective effect of quercetin on the morphology of pancreatic beta-cells of streptozotocin-treated diabetic rats," African Journal of Traditional, Complementary, and Alternative Medicines, vol. 4, pp. 64-74, 2006.

[82] E. Dokumacioglu, H. Iskender, T. M. Sen et al., "The effects of hesperidin and quercetin on serum tumor necrosis factoralpha and interleukin-6 levels in streptozotocin-induced diabetes model," Pharmacognosy Magazine, vol. 14, no. 54, pp. 167-173, 2018.

[83] R. M. Maciel, M. M. Costa, D. B. Martins et al., "Antioxidant and anti-inflammatory effects of quercetin in functional and morphological alterations in streptozotocin-induced diabetic rats," Research in Veterinary Science, vol. 95, no. 2, pp. 389397, 2013.

[84] I. C. Chis, A. Muresan, A. Oros, A. L. Nagy, and S. Clichici, "Protective effects of quercetin and chronic moderate exercise (training) against oxidative stress in the liver tissue of streptozotocin-induced diabetic rats," Acta Physiologica Hungarica, vol. 103, no. 1, pp. 49-64, 2016.

[85] H. Iskender, E. Dokumacioglu, T. M. Sen, I. Ince, Y. Kanbay, and S. Saral, "The effect of hesperidin and quercetin on oxidative stress, NF- $\kappa$ B and SIRT1 levels in a STZ-induced experimental diabetes model," Biomedicine \& Pharmacotherapy, vol. 90, pp. 500-508, 2017.

[86] O. O. Daniel, A. O. Adeoye, J. Ojowu, and O. O. Olorunsogo, "Inhibition of liver mitochondrial membrane permeability 
transition pore opening by quercetin and vitamin $\mathrm{E}$ in streptozotocin-induced diabetic rats," Biochemical and Biophysical Research Communications, vol. 504, no. 2, pp. 460469, 2018.

[87] Y. Zhang, H. Dong, M. Wang, and J. Zhang, "Quercetin isolated from Toona sinensis leaves attenuates hyperglycemia and protects hepatocytes in high-carbohydrate/high-fat diet and alloxan induced experimental diabetic mice," Journal Diabetes Research, vol. 2016, article 8492780, 10 pages, 2016.

[88] L. López, O. Varea, S. Navarro et al., "Benzbromarone, quercetin, and folic acid inhibit amylin aggregation," International Journal of Molecular Sciences, vol. 17, no. 6, p. 964, 2016.

[89] M. Kanter, C. Aktas, and M. Erboga, "Protective effects of quercetin against apoptosis and oxidative stress in streptozotocin-induced diabetic rat testis," Food and Chemical Toxicology, vol. 50, no. 3-4, pp. 719-725, 2012.

[90] C. F. Lin, Y. T. Kuo, T. Y. Chen, and C. T. Chien, "Quercetinrich guava (Psidium guajava) juice in combination with trehalose reduces autophagy, apoptosis and pyroptosis formation in the kidney and pancreas of type II diabetic rats," Molecules, vol. 21, no. 3, p. 334, 2016.

[91] N. Suganya, K. P. Mani, D. Sireesh et al., "Establishment of pancreatic microenvironment model of ER stress: quercetin attenuates $\beta$-cell apoptosis by invoking nitric oxide-cGMP signaling in endothelial cells," The Journal of Nutritional Biochemistry, vol. 55, pp. 142-156, 2018.

[92] X. Cai, L. Bao, X. Dai, Y. Ding, Z. Zhang, and Y. Li, "Quercetin protects RAW264.7 macrophages from glucosamineinduced apoptosis and lipid accumulation via the endoplasmic reticulum stress pathway," Molecular Medicine Reports, vol. 12, no. 5, pp. 7545-7553, 2015.

[93] X. Cai, L. Bao, Y. Ding, X. Dai, Z. Zhang, and Y. Li, "Quercetin alleviates cell apoptosis and inflammation via the ER stress pathway in vascular endothelial cells cultured in high concentrations of glucosamine," Molecular Medicine Reports, vol. 15, no. 2, pp. 825-832, 2017.

[94] S.-Y. Chen, C.-C. Chu, Y.-C. Lin, and P.-D. Duh, "Djulis (Chenopodium formosanum) and its bioactive compounds for management of hyperlipidemia and hyperglycemia in high-fat diet-fed mice," Journal of Food and Nutrition Research, vol. 7, no. 6, pp. 452-457, 2019.

[95] K. Sikder, N. Das, S. B. Kesh, and S. Dey, "Quercetin and $\beta$ sitosterol prevent high fat diet induced dyslipidemia and hepatotoxicity in Swiss albino mice," Indian Journal of Experimental Biology, vol. 52, no. 1, pp. 60-66, 2014.

[96] A. D. Mariee, G. M. Abd-Allah, and H. A. El-Beshbishy, "Protective effect of dietary flavonoid quercetin against lipemic-oxidative hepatic injury in hypercholesterolemic rats," Pharmaceutical Biology, vol. 50, no. 8, pp. 1019-1025, 2012.

[97] S. Juźwiak, J. Wójcicki, K. Mokrzycki et al., "Effect of quercetin on experimental hyperlipidemia and atherosclerosis in rabbits," Pharmacological Reports, vol. 57, no. 5, pp. 604609, 2005.

[98] J. B. S. Braun, J. B. Ruchel, S. A. Adefegha et al., "Neuroprotective effects of pretreatment with quercetin as assessed by acetylcholinesterase assay and behavioral testing in poloxamer-407 induced hyperlipidemic rats," Biomedicine \& Pharmacotherapy, vol. 88, pp. 1054-1063, 2017.

[99] E. Ulasova, J. Perez, B. G. Hill et al., "Quercetin prevents left ventricular hypertrophy in the Apo E knockout mouse," Redox Biology, vol. 1, no. 1, pp. 381-386, 2013.
[100] Q. H. Hu, X. Zhang, Y. Pan, Y. C. Li, and L. D. Kong, "Allopurinol, quercetin and rutin ameliorate renal NLRP3 inflammasome activation and lipid accumulation in fructose-fed rats," Biochemical Pharmacology, vol. 84, no. 1, pp. 113-125, 2012.

[101] M. Nekohashi, M. Ogawa, T. Ogihara et al., "Luteolin and quercetin affect the cholesterol absorption mediated by epithelial cholesterol transporter Niemann-Pick c1-like 1 in caco-2 cells and rats," PLoS One, vol. 9, no. 5, article e97901, 2014.

[102] C. H. Jung, I. Cho, J. Ahn, T. I. Jeon, and T. Y. Ha, "Quercetin reduces high-fat diet-induced fat accumulation in the liver by regulating lipid metabolism genes," Phytotherapy Research, vol. 27, no. 1, pp. 139-143, 2013.

[103] M. Kobori, S. Masumoto, Y. Akimoto, and H. Oike, "Chronic dietary intake of quercetin alleviates hepatic fat accumulation associated with consumption of a Western-style diet in C57/BL6J mice," Molecular Nutrition \& Food Research, vol. 55, no. 4, pp. 530-540, 2011.

[104] S. M. Lee, J. Moon, Y. Cho, J. H. Chung, and M. J. Shin, "Quercetin up-regulates expressions of peroxisome proliferator-activated receptor $\gamma$, liver $\mathrm{X}$ receptor $\alpha$, and ATP binding cassette transporter Al genes and increases cholesterol efflux in human macrophage cell line," Nutrition Research, vol. 33, no. 2, pp. 136-143, 2013.

[105] S. M. Ragab, S. Abd Elghaffar, T. H. el-Metwally, G. Badr, M. H. Mahmoud, and H. M. Omar, "Effect of a high fat, high sucrose diet on the promotion of non-alcoholic fatty liver disease in male rats: the ameliorative role of three natural compounds," Lipids in Health and Disease, vol. 14, no. 1, 2015.

[106] S. Krishna Mohan and M. Jainu, "Effect of pioglitazone, quercetin, and hydroxy citric acid on the lipid profile and lipoproteins in experimentally induced non-alcoholic steatohepatitis (NASH)," Indian Journal of Pharmaceutical Education and Research, vol. 48, no. 1, pp. 32-38, 2014.

[107] X. Zhu, T. Xiong, P. Liu et al., "Quercetin ameliorates HFDinduced NAFLD by promoting hepatic VLDL assembly and lipophagy via the IRE1a/XBP1s pathway," Food and Chemical Toxicology, vol. 114, pp. 52-60, 2018.

[108] S. Pisonero-Vaquero, Á. Martínez-Ferreras, M. V. GarcíaMediavilla et al., "Quercetin ameliorates dysregulation of lipid metabolism genes via the PI3K/AKT pathway in a diet-induced mouse model of nonalcoholic fatty liver disease," Molecular Nutrition \& Food Research, vol. 59, no. 5, pp. 879-893, 2015.

[109] X. Li, R. Wang, N. Zhou et al., "Quercetin improves insulin resistance and hepatic lipid accumulation in vitro in a NAFLD cell model," Biomedical Reports, vol. 1, no. 1, pp. 71-76, 2013.

[110] P. Liu, H. Lin, Y. Xu et al., "Frataxin-mediated PINK1Parkin-dependent mitophagy in hepatic steatosis: the protective effects of quercetin," Molecular Nutrition \& Food Research, vol. 62, no. 16, article e1800164, 2018.

[111] L. Liu, C. Gao, P. Yao, and Z. Gong, "Quercetin alleviates high-fat diet-induced oxidized low-density lipoprotein accumulation in the liver: implication for autophagy regulation," BioMed Research International, vol. 2015, Article ID 607531, 9 pages, 2015.

[112] H. Yang, T. Yang, C. Heng et al., "Quercetin improves nonalcoholic fatty liver by ameliorating inflammation, oxidative stress, and lipid metabolism in $\mathrm{db} / \mathrm{db}$ mice," Phytotherapy Research, vol. 33, no. 12, pp. 3140-3152, 2019. 
[113] S. K. Mohan and M. Jainu, "Effect of pioglitazone, quercetin and hydroxy citric acid on inflammatory markers in experimentally induced non alcoholic steatohepatitis (NASH)," Indian Journal of Pharmaceutical Education and Research, vol. 49, no. 2, pp. 152-157, 2015.

[114] H. Z. Ying, Y. H. Liu, B. Yu, Z. Y. Wang, J. N. Zang, and C. H. $\mathrm{Yu}$, "Dietary quercetin ameliorates nonalcoholic steatohepatitis induced by a high-fat diet in gerbils," Food and Chemical Toxicology, vol. 52, pp. 53-60, 2013.

[115] E. Marcolin, B. San-Miguel, D. Vallejo et al., "Quercetin treatment ameliorates inflammation and fibrosis in mice with nonalcoholic steatohepatitis," The Journal of Nutrition, vol. 142, no. 10, pp. 1821-1828, 2012.

[116] K. M. Surapaneni and M. Jainu, "Comparative effect of pioglitazone, quercetin and hydroxy citric acid on the status of lipid peroxidation and antioxidants in experimental nonalcoholic steatohepatitis," Journal of Physiology and Pharmacology, vol. 65, no. 1, pp. 67-74, 2014.

[117] K. M. Surapaneni, V. V. Priya, and J. Mallika, "Pioglitazone, quercetin and hydroxy citric acid effect on cytochrome P450 2E1 (CYP2E1) enzyme levels in experimentally induced non alcoholic steatohepatitis (NASH)," European Review for Medical and Pharmacological Sciences, vol. 18, no. 18, pp. 2736-2741, 2014.

[118] Q. Li, C. Zhao, Y. Zhang et al., "1H-NMR-based metabolomics coupled with molecular docking reveal the antidiabetic effects and potential active components of berberis vernae on type 2 diabetic rats," Frontiers in Pharmacology, vol. 11, p. 932, 2020.

[119] Y. Xu, J. Han, J. Dong et al., "Metabolomics characterizes the effects and mechanisms of quercetin in nonalcoholic fatty liver disease development," International Journal of Molecular Sciences, vol. 20, no. 5, p. 1220, 2019.

[120] A. A. Kolodziejczyk, D. Zheng, O. Shibolet, and E. Elinav, "The role of the microbiome in NAFLD and NASH," EMBO Molecular Medicine, vol. 11, no. 2, article e9302, 2019.

[121] C. Leung, L. Rivera, J. B. Furness, and P. W. Angus, "The role of the gut microbiota in NAFLD," Nature Reviews. Gastroenterology \& Hepatology, vol. 13, no. 7, pp. 412-425, 2016.

[122] H. Chu, Y. Duan, L. Yang, and B. Schnabl, "Small metabolites, possible big changes: a microbiota-centered view of non-alcoholic fatty liver disease," Gut, vol. 68, no. 2, pp. 359-370, 2019.

[123] D. Porras, E. Nistal, S. Martínez-Flórez et al., "Protective effect of quercetin on high-fat diet-induced non-alcoholic fatty liver disease in mice is mediated by modulating intestinal microbiota imbalance and related gut-liver axis activation," Free Radical Biology \& Medicine, vol. 102, pp. 188202, 2017.

[124] D. Porras, E. Nistal, S. Martínez-Flórez et al., "Functional interactions between gut microbiota transplantation, quercetin, and high-fat diet determine non-alcoholic fatty liver disease development in Germ-Free mice," Molecular Nutrition \& Food Research, vol. 63, no. 8, article e1800930, 2019.

[125] P. D. Petrov, M. V. García-Mediavilla, C. Guzmán et al., “A network involving gut microbiota, circulating bile acids, and hepatic metabolism genes that protects against nonalcoholic fatty liver disease," Molecular Nutrition \& Food Research, vol. 63, no. 20, article e1900487, 2019.

[126] P. Knekt, J. Kumpulainen, R. Järvinen et al., "Flavonoid intake and risk of chronic diseases," The American Journal of Clinical Nutrition, vol. 76, no. 3, pp. 560-568, 2002.
[127] M. D. G. van den Eynde, J. M. Geleijnse, J. Scheijen et al., "Quercetin, but not epicatechin, decreases plasma concentrations of methylglyoxal in adults in a randomized, doubleblind, placebo-controlled, crossover trial with pure flavonoids," The Journal of Nutrition, vol. 148, no. 12, pp. 19111916, 2018.

[128] S. A. Hussain, Z. A. Ahmed, T. O. Mahwi, and T. A. Aziz, "Quercetin dampens postprandial hyperglycemia in type 2 diabetic patients challenged with carbohydrates load," International Journal of Diabetes Research, vol. 1, no. 3, pp. 3235, 2012.

[129] Z. Mazloom, S. M. Abdollahzadeh, M.-H. Dabbaghmanesh, and A. Rezaianzadeh, "The effect of quercetin supplementation on oxidative stress, glycemic control, lipid profile and insulin resistance in type 2 diabetes: a randomized clinical trial," Journal of Health Sciences \& Surveillance System, vol. 2, pp. 8-14, 2014.

[130] V. Ostadmohammadi, A. Milajerdi, E. Ayati, F. Kolahdooz, and Z. Asemi, "Effects of quercetin supplementation on glycemic control among patients with metabolic syndrome and related disorders: a systematic review and meta-analysis of randomized controlled trials," Phytotherapy Research, vol. 33, no. 5, pp. 1330-1340, 2019.

[131] T. M. Lu, H. F. Chiu, Y. C. Shen, C. C. Chung, K. Venkatakrishnan, and C. K. Wang, "Hypocholesterolemic efficacy of quercetin rich onion juice in healthy mild hypercholesterolemic adults: a pilot study," Plant Foods for Human Nutrition, vol. 70, no. 4, pp. 395-400, 2015.

[132] Y. Pasdar, F. Oubari, M. N. Zarif, M. Abbasi, A. Pourmahmoudi, and M. Hosseinikia, "Effects of quercetin supplementation on hematological parameters in nonalcoholic fatty liver disease: a randomized, double-blind, placebo-controlled pilot study," Clinical Nutrition Research, vol. 9, no. 1, pp. 11-19, 2020.

[133] B. Salehi, L. Machin, L. Monzote et al., "Therapeutic potential of quercetin: new insights and perspectives for human health," ACS Omega, vol. 5, no. 20, pp. 1184911872, 2020.

[134] J. Singh, P. Mittal, G. Vasant Bonde, G. Ajmal, and B. Mishra, "Design, optimization, characterization and in-vivo evaluation of quercetin enveloped Soluplus ${ }^{\circledR} / \mathrm{P} 407$ micelles in diabetes treatment," Artificial Cells, Nanomedicine, and Biotechnology, vol. 46, no. sup3, pp. S546-S555, 2018.

[135] A. Riva, A. Corti, G. Belcaro et al., "Interaction study between antiplatelet agents, anticoagulants, diabetic therapy and a novel delivery form of quercetin," Minerva Cardioangiologica, vol. 67, no. 1, pp. 79-83, 2019.

[136] A. P. Singh, R. Singh, S. S. Verma et al., "Health benefits of resveratrol: evidence from clinical studies," Medicinal Research Reviews, vol. 39, no. 5, pp. 1851-1891, 2019.

[137] Y. Fan and O. Pedersen, "Gut microbiota in human metabolic health and disease," Nature Reviews. Microbiology, vol. 19, no. 1, pp. 55-71, 2021.

[138] J. Xu, H. B. Chen, and S. L. Li, "Understanding the molecular mechanisms of the interplay between herbal medicines and gut microbiota," Medicinal Research Reviews, vol. 37, no. 5, pp. 1140-1185, 2017.

[139] J. Xie, W. Song, X. Liang et al., "Protective effect of quercetin on streptozotocin-induced diabetic peripheral neuropathy rats through modulating gut microbiota and reactive oxygen species level," Biomedicine \& Pharmacotherapy, vol. 127, p. $110147,2020$. 